Hautarzt 2020 71 (Suppl 1):S1

https://doi.org/10.1007/s00105-020-04650-y

(c) Springer Medizin Verlag GmbH, ein Teil von Springer Nature 2020

Liebe Kolleginnen und Kollegen,

wir freuen uns, Ihnen auch im Rahmen unserer diesjährigen digitalen Fortbildungswoche eine DIA-KLINIK bieten zu können - und das nicht nur in Form der von Ihnen so geschätzten Vorträge, die Sie diesmal bequem vom eigenen Arbeitsplatz oder von zu Hause aus abrufen können, sondern auch in Form dieses Sonderheftes der Zeitschrift Der Hautarzt, das Sie jetzt in den Händen halten.

Gerade in der jetzigen Situation ist die Erstellung der DIA-KLINIKeine Herausforderung, die nur im Team zu meistern ist. Allen voran möchten wir den Assistentinnen und Assistenten unserer Klinik danken, mit denen wir wieder spannende und lehrreiche Fälle aus den vergangenen zwei Jahren für Sie aufbereiten konnten. Diagnostik, Therapie und Dokumentation außergewöhnlicher Krankheitsfälle sind auch nur durch die Mitwirkung und das exzellente Können aller Mitarbeiter der Klinik, insbesondere des Fotolaborteams, der medizinisch-technischen Angestellten in allen Laborbereichen und den Pflegekräften möglich.

Die Zusammenarbeit mit Frau Kind und ihrem Team vom Springer Medizin Verlag, die uns von der Manuskripterstellung bis zur Drucklegung unermüdlich

Elke C. Sattler · Laura Engels · Eva Maria Oppel · Matthias Betke · Michael J. Flaig • Lars E. French

Klinik und Poliklinik für Dermatologie und Allergologie, Klinikum der Universität München, LMU München, München, Deutschland

\title{
Vorwort zur DIA-KLINIK der 27. Fortbildungswoche für praktische Dermatologie und Venerologie FOBI digital 2020
}

und tatkräftig begleiten, wissen wir sehr zu schätzen. Für die finanzielle Unterstützung dieses Sonderheftes danken wir der LEO Pharma GmbH.

Unser größter Dank gilt aber unseren Patientinnen und Patienten, die es uns durch ihr entgegengebrachtes Vertrauen und ihr Einverständnis zur Veröffentlichung ermöglichen, wertvolle Erfahrungen und Erkenntnisse weiterzugeben.

Freuen Sie sich, unsere geschätzten Kolleginnen und Kollegen, auf eine Mischung aus hochkarätigen Kasuistiken aus allen Teilbereichen der Dermatologie: von Allergologie über Genetik, Dermatopathologie und Immunologie zu Onkologie und Systemerkrankungen - von alltäglich anmutenden Anamnesen, die dann doch eine interessante Wendung nahmen, von seltenen Differenzialdiagnosen, Chamäleons und altbekanntem Wissen zu spannendem Wiederentdecktem und Neuem!

Viel Spaß beim Lesen!

Elke C. Sattler

Laura Engels

Eva Maria Oppel

Matthias Betke

Michael J. Flaig

Lars E. French

München, den 18.06.2020

\section{Korrespondenzadresse}

Prof. Dr. med. Elke C. Sattler

Klinik und Poliklinik für Dermatologie und Allergologie, Klinikum der Universität München, LMU München

Frauenlobstr. 9-11, 80337 München,

Deutschland

Elke.Sattler@med.uni-muenchen.de

Interessenkonflikt. E.C. Sattler, L. Engels, E.M. Oppel, M. Betke, M.J. Flaig und L.E. French geben an, dass kein Interessenkonflikt besteht.

This article is part of a supplement sponsored by LEO Pharma GmbH 